\title{
Linear Size-structured Population Models with Spacial Diffusion and Optimal Harvesting Problems
}

\author{
N. Kato ${ }^{1} *$ \\ ${ }^{1}$ Faculty of Electrical and Computer Engineering, Institute of Science and Engineering \\ Kanazawa University, Kakuma-machi, Kanazawa, 920-1192, Japan
}

\begin{abstract}
We first investigate linear size-structured population models with spacial diffusion. Existence of a unique mild solution is established. Then we consider a harvesting problem for linear size-structured models with diffusion and show the existence of an optimal harvesting effort to maximize the total price or total harvest.
\end{abstract}

Keywords and phrases: size-structured, evolution population models, mild solutions, optimal harvesting

Mathematics Subject Classification: 35Q92, 47D06, 49J20, 92D25

\section{Introduction}

We are concerned with linear size-structured population models with spacial diffusion. Size is recognized as an important variable to describe some kinds of population dynamics. For example, to describe population dynamics of plants or fishes, it is known that size is better than age. See [5] for an introduction and models of size-structured population without diffusion. Age-structured population models with diffusion have been investigated by many authors as seen in [2, Chap. 4] and the references therein. See also [12] for semigroup approach on steady states. On the other hand, for size-structured population models, only the case without diffusion has been studied so far. See e.g. Calsina-Saldaña [3], Ackleh et al. [1], and Kato [8-10] for existence results, Farkas and Hagen [6] for stability and regularity results. In addition, Hritonenko et al. [7] have considered optimal harvesting problem for plant management by using size-structured models. We also mention that Webb [14] has studied population models structured by age, size and spacial position, where the birth process is determined by age, not by size.

Let us consider a biological population living in a habitat $\Omega \subset \mathbb{R}^{d}(d=1,2,3)$ which is a bounded domain with smooth boundary $\partial \Omega$. Let $p(s, t, x)$ be the population density with respect to size $s \in\left[0, s_{\dagger}\right)$ and position $x \in \Omega$ at time $t \in[0, T]$, where $s_{\dagger} \in(0, \infty)$ is the maximal size, $T \in(0, \infty)$ is a given time. We assume that the growth rate $g(s, t)$ depends on size $s$ and time $t$ and independent of position $x$, while the fertility rate $\beta(s, t, x)$ and the mortality rate $\mu(s, t, x)$ depend on size $s$, time $t$ and position $x$. Throughout the paper, we set $\Omega_{T}:=(0, T) \times \Omega, Q:=\left(0, s_{\dagger}\right) \times \Omega, \mathcal{Q}_{T}:=\left(0, s_{\dagger}\right) \times(0, T) \times \Omega$ and

${ }^{*}$ Corresponding author. E-mail: nkato@se.kanazawa-u.ac.jp 
$\Sigma_{T}:=\left(0, s_{\dagger}\right) \times(0, T) \times \partial \Omega$. We consider the following harvesting problem with harvesting effort $u(s, t, x)$ :

$$
\left.\begin{array}{ll}
\partial_{t} p(s, t, x)+\partial_{s}(g(s, t) p(s, t, x))-\Delta p(s, t, x) & \\
\quad=-\mu(s, t, x) p(s, t, x)-u(s, t, x) p(s, t, x)+f(s, t, x) & \text { in } \mathcal{Q}_{T}, \\
g(0, t) p(0, t, x)=C(t, x)+\int_{0}^{s_{\dagger}} \beta(s, t, x) p(s, t, x) d s & \text { in } \Omega_{T}, \\
\frac{\partial p}{\partial \nu}(s, t, x)=0 & \text { on } \Sigma_{T}, \\
p(s, 0, x)=p_{0}(s, x) & \text { in } Q,
\end{array}\right\}
$$

where $\Delta$ is the Laplacian, $f(s, t, x)$ and $C(t, x)$ represent inflows of $s$-size and zero-size populations respectively from outside, and $p_{0}$ is a given initial data. The Neumann boundary condition in the third equation means the individuals never go out from the habitat $\Omega$, where $\partial / \partial \nu$ stands for the outward normal derivative. The harvesting effort $u$ is assumed to belong to the control set

$$
\mathcal{U}=\left\{u \in L^{\infty}\left(\mathcal{Q}_{T}\right) \mid \zeta_{1}(s, t, x) \leq u(s, t, x) \leq \zeta_{2}(s, t, x) \quad \text { a.e. }(s, t, x) \in \mathcal{Q}_{T}\right\}
$$

where $\zeta_{1}, \zeta_{2} \in L^{\infty}\left(\mathcal{Q}_{T}\right), 0 \leq \zeta_{1}(s, t, x) \leq \zeta_{2}(s, t, x)$ a.e. in $\mathcal{Q}_{T}$. In this paper, we employ a mild solution introduced by using characteristic method and semigroup theory. See Definition 2.1 below. Denoting by $p^{u}$ the mild solution of (1.1) corresponding to the harvesting effort $u \in \mathcal{U}$, we consider the following optimal harvesting problem:

$$
\text { Maximize } \int_{\mathcal{Q}_{T}} w(s, t, x) u(s, t, x) p^{u}(s, t, x) d s d t d x \quad \text { subject to } u \in \mathcal{U},
$$

where $w(s, t, x)$ is a given weight function. Such a problem without diffusion has been studied in Kato [11]. The integral in (1.2) represents the total price, or total amount of harvest if $w \equiv 1$. In Anita [2], similar optimal harvesting problem has been studied for age-structured models with diffusion. There, the solution of the model is formulated along characteristic line through the theory of heat equation.

In Section 2, we investigate the existence results to the harvesting problem (1.1). For that purpose, since $\mu+u$ works as a mortality rate, it suffices to consider the case without harvesting effort. See Corollary 2.5 below. Then we show the existence of an optimal control to the above optimal harvesting problem (1.2) governed by (1.1) in Section 3.

\section{Existence of solutions}

We first consider the following linear size-structured population model with diffusion:

$$
\left.\begin{array}{ll}
\partial_{t} p(s, t, x)+\partial_{s}(g(s, t) p(s, t, x))-\Delta p(s, t, x)=-\mu(s, t, x) p(s, t, x)+f(s, t, x) & \text { in } \mathcal{Q}_{T}, \\
g(0, t) p(0, t, x)=C(t, x)+\int_{0}^{s_{\dagger}} \beta(s, t, x) p(s, t, x) d s & \text { in } \Omega_{T}, \\
\frac{\partial p}{\partial \nu}(s, t, x)=0 & \text { on } \Sigma_{T}, \\
p(s, 0, x)=p_{0}(s, x) & \text { in } Q,
\end{array}\right\}
$$

We assume the following assumptions:

(H1) $g:\left[0, s_{\dagger}\right] \times[0, T] \rightarrow[0, \infty)$ is continuously differentiable and $\left|\partial_{s} g(s, t)\right| \leq L_{g}$ for some constant $L_{g}>0$, $g(s, t)>0$ if $s \in\left[0, s_{\dagger}\right)$ and $g\left(s_{\dagger}, t\right)=0$.

(H2) $\mu, \beta \in L^{\infty}\left(\mathcal{Q}_{T}\right)$ and $\mu(s, t, x) \geq 0, \beta(s, t, x) \geq 0$ a.e.

(H3) $f \in L^{2}\left(\mathcal{Q}_{T}\right), f(s, t, x) \geq 0$ a.e. in $\mathcal{Q}_{T} . C \in L^{2}\left(\Omega_{T}\right), C(t, x) \geq 0$ a.e. in $\Omega_{T} \cdot p_{0} \in L^{2}(Q), p_{0}(s, x) \geq 0$ a.e. in $Q$. 
We extend $g$ on $\mathbb{R} \times[0, T]$ by putting $g(s, t)=g(0, t)$ for $s<0$ and $g(s, t)=0$ for $s>s_{\dagger}$. Note that the extended $g(s, t)$ satisfies the Lipschitz condition:

$$
\left|g\left(s_{1}, t\right)-g\left(s_{2}, t\right)\right| \leq L_{g}\left|s_{1}-s_{2}\right|, \quad \forall s_{1}, s_{2} \in \mathbb{R}, t \in[0, T] .
$$

We define the characteristic curve $\varphi\left(t ; t_{0}, s_{0}\right)$ through $\left(s_{0}, t_{0}\right) \in \mathbb{R} \times[0, T]$ by the unique solution of the following differential equation

$$
s^{\prime}(t)=g(s(t), t), t \in[0, T], \quad s\left(t_{0}\right)=s_{0} .
$$

Let $z(t):=\varphi(t ; 0,0)$. For $(s, t) \in\left[0, s_{\dagger}\right) \times[0, T]$ satisfying $s<z(t)$, define $\tau:=\tau(t, s)$ by the relation

$$
\varphi(t ; \tau, 0)=s, \text { or equivalently, } \varphi(\tau ; t, s)=0 .
$$

Then define the initial time $\tau^{*}(t, s)$ for $(s, t) \in\left[0, s_{\dagger}\right) \times[0, T]$ by

$$
\tau^{*}(t, s)= \begin{cases}\tau(t, s) & s<z(t) \\ 0 & s \geq z(t)\end{cases}
$$

For $\gamma \in L^{\infty}\left(\left(0, s_{\dagger}\right) \times(0, T)\right)$, we set

$$
\Pi_{\gamma}\left(t, \tau, t_{0}, s_{0}\right)=\exp \left(\int_{\tau}^{t} \gamma\left(\varphi\left(\sigma ; t_{0}, s_{0}\right), \sigma\right) d \sigma\right)
$$

for $\left(s_{0}, t_{0}\right) \in\left[0, s_{\dagger}\right) \times[0, T]$.

Let $A$ be the realization of Laplacian $\Delta$ in $L^{2}(\Omega)$ with the Neumann boundary condition, i.e.,

$$
\begin{aligned}
& D(A)=\left\{\phi \in H^{2}(\Omega) \mid \frac{\partial \phi}{\partial \nu}(x)=0 \quad \text { a.e. on } \partial \Omega\right\} \subset L^{2}(\Omega) \\
& A \phi=\Delta \phi \text { for } \phi \in D(A) .
\end{aligned}
$$

It is known that $A$ generates an analytic semigroup $\{T(t) \mid t \geq 0\}$ in $L^{2}(\Omega)$. See e.g. [4]. We translate the problem (2.1) to the following abstract problem in $L^{2}(\Omega)$ :

$$
\left.\begin{array}{ll}
\partial_{t} p(s, t)+\partial_{s}(g(s, t) p(s, t))-A p(s, t)=G(t, p(\cdot, t))(s), & s \in\left[0, s_{\dagger}\right), t \in[0, T], \\
g(0, t) p(0, t)=F(t, p(\cdot, t)), & t \in[0, T], \\
p(s, 0)=p_{0}(s), & s \in\left[0, s_{\dagger}\right),
\end{array}\right\}
$$

where $p(s, t)$ is an $L^{2}(\Omega)$-valued function and

$$
\begin{aligned}
& {[F(t, p(\cdot, t))](x):=C(t, x)+\int_{0}^{s_{\dagger}} \beta(s, t, x) p(s, t, x) d s,} \\
& {[G(t, p(\cdot, t))(s)](x):=-\mu(s, t, x) p(s, t, x)+f(s, t, x) .}
\end{aligned}
$$

Note that $F(t, p(\cdot, t)) \in L^{2}(\Omega)$ and $G(t, p(\cdot, t))(s) \in L^{2}(\Omega)$. Similar abstraction was done in Webb [13] for age-structured models without diffusion.

Definition 2.1. A function $p \in L^{\infty}\left(0, T ; L^{2}(Q)\right)$ is said to be a mild solution to (2.1) if $p$ satisfies

$$
p(s, t)=\left\{\begin{array}{c}
T(t-\tau) \Pi_{\gamma}(t, \tau ; t, s) \frac{F(\tau, p(\cdot, \tau))}{g(0, \tau)} \\
\quad+\int_{\tau}^{t} T(t-\sigma) \Pi_{\gamma}(t, \sigma ; t, s) G(\sigma, p(\cdot, \sigma))(\varphi(\sigma ; t, s)) d \sigma \\
T(t) \Pi_{\gamma}(t, 0 ; t, s) p_{0}(\varphi(0 ; t, s)) \\
\quad+\int_{0}^{t} T(t-\sigma) \Pi_{\gamma}(t, \sigma ; t, s) G(\sigma, p(\cdot, \sigma))(\varphi(\sigma ; t, s)) d \sigma \\
\quad \text { a.e. } s \in\left(z(t), s_{\dagger}\right),
\end{array}\right.
$$


where $\tau:=\tau(t, s)$ is defined by $(2.2)$ and $\gamma(s, t):=-\partial_{s} g(s, t)$.

Remark 2.2. Note that each function $p \in L^{\infty}\left(0, T ; L^{2}(Q)\right)$ can be regarded as a function in $L^{2}\left(\left(0, s_{\dagger}\right) \times\right.$ $\left.(0, T) ; L^{2}(\Omega)\right)$ as well as a function in $L^{2}\left(\mathcal{Q}_{T}\right)$ by the relation

$$
[p(t)](s, x)=[p(s, t)](x)=p(s, t, x) \quad \text { for a.e. }(s, t, x) \in \mathcal{Q}_{T}:=\left(0, s_{\dagger}\right) \times(0, T) \times \Omega .
$$

The following proposition states the relation between mild solutions and the problem (2.1).

Proposition 2.3. Let $p \in L^{\infty}\left(0, T ; L^{2}(Q)\right)$ be a mild solution to $(2.1)$. Then for a.e. $\left(s_{0}, t_{0}\right) \in\left(0, s_{\dagger}\right) \times$ $\{0\} \cup\{0\} \times(0, T)$, the function $t \mapsto p\left(\varphi\left(t ; t_{0}, s_{0}\right), \eta\right)$ is continuously differentiable from $\left[\tau^{*}\left(t_{0}, s_{0}\right), T\right]$ into $L^{2}(\Omega)$ and satisfies (2.4), and so (2.1) as well, along characteristic curves, where $\tau^{*}\left(t_{0}, s_{0}\right)$ appears in $(2.3)$.

Proof. For a.e. $t_{0} \in(0, T)$, since $\tau^{*}\left(t_{0}, 0\right)=\tau\left(t_{0}, 0\right)=t_{0}$ and $\varphi\left(t ; t_{0}, 0\right)<z(t)$ for $t \in\left(t_{0}, T\right)$, it follows from (2.7) that

$$
\begin{aligned}
p\left(\varphi\left(t ; t_{0}, 0\right), t\right)= & T\left(t-t_{0}\right) \Pi_{\gamma}\left(t, t_{0} ; t_{0}, 0\right) \frac{F\left(t_{0}, p\left(\cdot, t_{0}\right)\right)}{g\left(0, t_{0}\right)} \\
& +\int_{t_{0}}^{t} T(t-\sigma) \Pi_{\gamma}\left(t, \sigma ; t_{0}, 0\right) G(\sigma, p(\cdot, \sigma))\left(\varphi\left(\sigma ; t_{0}, 0\right)\right) d \sigma .
\end{aligned}
$$

Since $\{T(t) \mid t \geq 0\}$ is an analytic semigroup, the right side of (2.8) is continuously differentiable in $t$ as a mapping from $\left(t_{0}, T\right)$ into $L^{2}(\Omega)$ and we have

$$
\begin{aligned}
\frac{d}{d t} p\left(\varphi\left(t ; t_{0}, 0\right), t\right)= & A p\left(\varphi\left(t ; t_{0}, 0\right), t\right)-\partial_{s} g\left(\varphi\left(t ; t_{0}, 0\right), t\right) p\left(\varphi\left(t ; t_{0}, 0\right), t\right) \\
& +G(t, p(\cdot, t))\left(\varphi\left(t ; t_{0}, 0\right)\right)
\end{aligned}
$$

For a.e. $s_{0} \in\left(0, s_{\dagger}\right)$, since $\tau^{*}\left(0, s_{0}\right)=0$ and $\varphi\left(t ; 0, s_{0}\right)>z(t)$ for $t \in(0, T)$, it follows from (2.7) that

$$
\begin{aligned}
p\left(\varphi\left(t ; 0, s_{0}\right), t\right)= & T(t) \Pi_{\gamma}\left(t, 0 ; 0, s_{0}\right) p_{0}\left(\varphi\left(0 ; 0, s_{0}\right)\right) \\
& +\int_{0}^{t} T(t-\sigma) \Pi_{\gamma}\left(t, \sigma ; 0, s_{0}\right) G(\sigma, p(\cdot, \sigma))\left(\varphi\left(\sigma ; 0, s_{0}\right)\right) d \sigma .
\end{aligned}
$$

Again, since $\{T(t) \mid t \geq 0\}$ is an analytic semigroup, the right side of (2.10) is continuously differentiable in $t$ as a mapping from $(0, T)$ into $L^{2}(\Omega)$ and we have

$$
\begin{aligned}
\frac{d}{d t} p\left(\varphi\left(t ; 0, s_{0}\right), t\right)= & A p\left(\varphi\left(t ; 0, s_{0}\right), t\right)-\partial_{s} g\left(\varphi\left(t ; 0, s_{0}\right), t\right) p\left(\varphi\left(t ; 0, s_{0}\right), t\right) \\
& +G(t, p(\cdot, t))\left(\varphi\left(t ; 0, s_{0}\right)\right) .
\end{aligned}
$$

Furthermore, it follows from (2.10) that $p$ satisfies the initial condition in the sense that

$$
p(s, 0):=\lim _{\eta \downarrow 0} p(\varphi(\eta ; 0, s), \eta)=p_{0}(s), \quad \text { a.e. } \quad s \in\left(0, s_{\dagger}\right) .
$$

(2.9), (2.11), and (2.12) show that the mild solution $p$ satisfies (2.4), and so (2.1), along characteristic curves.

Let $\alpha \in \mathbb{R}$. For positivity of mild solutions, we rewrite (2.4) by adding $\alpha p(s, t)$ to both sides of the first equation. Then mild solution $p \in L^{\infty}\left(0, T ; L^{2}(Q)\right)$ is rewritten as the following form as well: For a.e. 
$t \in(0, T)$,

$$
p(s, t)=\left\{\begin{array}{l}
T(t-\tau) \Pi_{\gamma_{\alpha}}(t, \tau ; t, s) \frac{F(\tau, p(\cdot, \tau))}{g(0, \tau)} \\
+\int_{\tau}^{t} T(t-\sigma) \Pi_{\gamma_{\alpha}}(t, \sigma ; t, s)[G+\alpha I](\sigma, p(\cdot, \sigma))(\varphi(\sigma ; t, s)) d \sigma \\
T(t) \Pi_{\gamma_{\alpha}}(t, 0 ; t, s) p_{0}(\varphi(0 ; t, s)) \\
+\int_{0}^{t} T(t-\sigma) \Pi_{\gamma_{\alpha}}(t, \sigma ; t, s)[G+\alpha I](\sigma, p(\cdot, \sigma))(\varphi(\sigma ; t, s)) d \sigma \\
\quad \text { a.e. } s \in\left(z(t), s_{\dagger}\right),
\end{array}\right.
$$

where $\tau:=\tau(t, s)$ and $\gamma_{\alpha}(s, t):=-\partial_{s} g(s, t)-\alpha$. Actually, it is shown that if $p \in L^{\infty}\left(0, T ; L^{2}(Q)\right)$ satisfies (2.13), then $p$ is a mild solution to (2.1) and the converse is also true. We omit the details. Concerning the existence of solutions, we have

Theorem 2.4. Let $(\mathrm{H} 1)-(\mathrm{H} 3)$ hold. Then there exists a unique mild solution $p \in L^{\infty}\left(0, T ; L^{2}(Q)\right)$ of (2.1), which satisfies $p(s, t, x) \geq 0$ a.e. $(s, t, x) \in \mathcal{Q}_{T}$ and

$$
\|p(\cdot, t)\|_{L^{2}(Q)}^{2} \leq \tilde{C}_{T}\left\{\left\|p_{0}\right\|_{L^{2}(Q)}^{2}+\|C\|_{L^{2}\left(\Omega_{T}\right)}^{2}+\|f\|_{L^{2}\left(\mathcal{Q}_{T}\right)}^{2}\right\}
$$

for some constant $\tilde{C}_{T}>0$ depending on $\|\beta\|_{L^{\infty}\left(\mathcal{Q}_{T}\right)},\|\mu\|_{L^{\infty}\left(\mathcal{Q}_{T}\right)}$ and $\underline{g}_{T}:=\min _{t \in[0, T]} g(0, t)>0$.

Proof. Set $E_{T,+}:=\left\{p \in L^{\infty}\left(0, T ; L^{2}(Q)\right) \mid p(s, t, x) \geq 0\right.$ a.e. $\left.(s, t, x) \in \mathcal{Q}_{T}\right\}$. Put $\alpha=\|\mu\|_{L^{\infty}\left(\mathcal{Q}_{T}\right)}$ and define the mapping $K_{\alpha}$ on $E_{T,+}$ by

$$
\left[K_{\alpha} p\right](s, t)=\left\{\begin{array}{l}
T(t-\tau) \Pi_{\gamma_{\alpha}}(t, \tau ; t, s) \frac{F(\tau, p(\cdot, \tau))}{g(0, \tau)} \\
+\int_{\tau}^{t} T(t-\sigma) \Pi_{\gamma_{\alpha}}(t, \sigma ; t, s)[G+\alpha I](\sigma, p(\cdot, \sigma))(\varphi(\sigma ; t, s)) d \sigma \\
T(t) \Pi_{\gamma_{\alpha}}(t, 0 ; t, s) p_{0}(\varphi(0 ; t, s)) \\
+\int_{0}^{t} T(t-\sigma) \Pi_{\gamma_{\alpha}}(t, \sigma ; t, s)[G+\alpha I](\sigma, p(\cdot, \sigma))(\varphi(\sigma ; t, s)) d \sigma \\
\quad \text { a.e. } s \in\left(z(t), s_{\dagger}\right),
\end{array}\right.
$$

where $\tau:=\tau(t, s)$ and $\gamma_{\alpha}(s, t):=-\partial_{s} g(s, t)-\alpha$. We will seek a fixed point of $K_{\alpha}$. To do this, we will show that $K_{\alpha}$ maps $E_{T,+}$ into itself and that $K_{\alpha}$ is a contraction mapping in $E_{T,+}$. In what follows, we put $F_{p}(t):=F(t, p(\cdot, t))$ and $G_{p, \alpha}(s, t):=[G+\alpha I](t, p(\cdot, t))(s)$ for simplicity of notation. First, note that $K_{\alpha} p(s, t) \in L^{2}(\Omega)$ and $\left[K_{\alpha} p(s, t)\right](x) \geq 0$ for $p \in E_{T,+}$. By definition of $K_{\alpha} p$, we have

$$
\begin{aligned}
\left\|K_{\alpha} p(\cdot, t)\right\|_{L^{2}(Q)}^{2} & =\int_{0}^{z(t)}\left\|K_{\alpha} p(s, t)\right\|_{L^{2}(\Omega)}^{2} d s+\int_{z(t)}^{s_{\dagger}}\left\|K_{\alpha} p(s, t)\right\|_{L^{2}(\Omega)}^{2} d s \\
& \leq K_{1}^{2}(t)+K_{2}^{2}(t)+K_{3}^{2}(t)+K_{4}^{2}(t),
\end{aligned}
$$

where

$$
K_{1}^{2}(t)=\int_{0}^{z(t)}\left\|T(t-\tau) \Pi_{\gamma_{\alpha}}(t, \tau ; t, s) \frac{F_{p}(\tau)}{g(0, \tau)}\right\|_{L^{2}(\Omega)}^{2} d s
$$




$$
\begin{gathered}
K_{2}^{2}(t)=\int_{0}^{z(t)}\left(\int_{\tau}^{t}\left\|T(t-\sigma) \Pi_{\gamma_{\alpha}}(t, \sigma ; t, s) G_{p, \alpha}(\varphi(\sigma ; t, s), \sigma)\right\|_{L^{2}(\Omega)} d \sigma\right)^{2} d s, \\
K_{3}^{2}(t)=\int_{z(t)}^{s_{\dagger}}\left\|T(t) \Pi_{\gamma_{\alpha}}(t, 0 ; t, s) p_{0}(\varphi(0 ; t, s))\right\|_{L^{2}(\Omega)}^{2} d s \\
K_{4}^{2}(t)=\int_{z(t)}^{s_{\dagger}}\left(\int_{0}^{t}\left\|T(t-\sigma) \Pi_{\gamma_{\alpha}}(t, \sigma ; t, s) G_{p, \alpha}(\varphi(\sigma ; t, s), \sigma)\right\|_{L^{2}(\Omega)} d \sigma\right)^{2} d s .
\end{gathered}
$$

Recall that the semigroup $\{T(t)\}$ satisfies $\|T(t) \phi\|_{L^{2}(\Omega)} \leq\|\phi\|_{L^{2}(\Omega)}$ for $\phi \in L^{2}(\Omega)$. For $K_{1}^{2}(t)$, we use change of variable from $s$ to $\sigma$ by $\sigma=\tau(t, s)$. Since $d s / d \sigma=-g(0, \sigma) \Pi_{\partial_{s} g}(t, \sigma ; t, s)=$ $-g(0, \sigma) \Pi_{\partial_{s} g}(t, \sigma ; \sigma, 0)$, we have

$$
\begin{aligned}
K_{1}^{2}(t) & =\int_{0}^{t}\left\|T(t-\sigma) F_{p}(\sigma)\right\|_{L^{2}(\Omega)}^{2} \frac{1}{g(0, \sigma)} \Pi_{2 \gamma_{\alpha}+\partial_{s} g}(t, \sigma ; \sigma, 0) d \sigma \\
& \leq \frac{1}{\underline{g}_{T}} e^{L_{g} T} \int_{0}^{t}\|F(\sigma, p(\cdot, \sigma))\|_{L^{2}(\Omega)}^{2} d \sigma
\end{aligned}
$$

where $\underline{g}_{T}:=\min _{t \in[0, T]} g(0, t)>0$. For $K_{3}^{2}(t)$, use change of variable from $s$ to $\xi$ by $\xi=\varphi(0 ; t, s)$. Since $d s / d \xi=\Pi_{\partial_{s} g}(t, 0, t, s)=\Pi_{\partial_{s} g}(t, 0,0, \xi)$, we obtain

$$
K_{3}^{2}(t)=\int_{0}^{s_{\dagger}}\left\|T(t) p_{0}(\xi)\right\|_{L^{2}(\Omega)}^{2} \Pi_{2 \gamma_{\alpha}+\partial_{s} g}(t, 0 ; 0, \xi) d \xi \leq e^{L_{g} T}\left\|p_{0}\right\|_{L^{2}(Q)}^{2}
$$

To estimate $K_{2}^{2}(t)+K_{4}^{2}(t)$, we use Schwarz's inequality, Fubini's theorem and change of variable from $s$ to $\xi$ by $\xi=\varphi(\sigma ; t, s)$. Noting that $d s / d \xi=\Pi_{\partial_{s} g}(t, \sigma, t, s)=\Pi_{\partial_{s} g}(t, \sigma, \sigma, \xi)$, we have

$$
\begin{aligned}
K_{2}^{2}(t)+K_{4}^{2}(t) \leq & T \int_{0}^{z(t)} \int_{\tau}^{t}\left\|T(t-\sigma) \Pi_{\gamma_{\alpha}}(t, \sigma ; t, s) G_{p, \alpha}(\varphi(\sigma ; t, s), \sigma)\right\|_{L^{2}(\Omega)}^{2} d \sigma d s \\
& +T \int_{z(t)}^{s_{\dagger}} \int_{0}^{t}\left\|T(t-\sigma) \Pi_{\gamma_{\alpha}}(t, \sigma ; t, s) G_{p, \alpha}(\varphi(\sigma ; t, s), \sigma)\right\|_{L^{2}(\Omega)}^{2} d \sigma d s \\
= & T \int_{0}^{t} \int_{\tau^{-1}(\sigma)}^{s_{\dagger}}\left\|T(t-\sigma) \Pi_{\gamma_{\alpha}}(t, \sigma ; t, s) G_{p, \alpha}(\varphi(\sigma ; t, s), \sigma)\right\|_{L^{2}(\Omega)}^{2} d s d \sigma \\
= & T \int_{0}^{t} \int_{0}^{s_{\dagger}}\left\|T(t-\sigma) G_{p, \alpha}(\xi, \sigma)\right\|_{L^{2}(\Omega)}^{2} \Pi_{2 \gamma_{\alpha}+\partial_{s} g}(t, \sigma ; \sigma, \xi) d \xi d \sigma \\
\leq & T e^{L_{g} T} \int_{0}^{t}\|G(\sigma, p(\cdot, \sigma))+\alpha p(\cdot, \sigma)\|_{L^{2}(Q)}^{2} d \sigma
\end{aligned}
$$

From (2.5), (2.6), and assumptions (H2), (H3), we find that

$$
\begin{gathered}
\|F(\sigma, p(\cdot, \sigma))\|_{L^{2}(\Omega)}^{2} \leq 2\left(\|C(\sigma, \cdot)\|_{L^{2}(\Omega)}^{2}+\|\beta\|_{L^{\infty}\left(\mathcal{Q}_{\mathcal{T}}\right)}^{2} s_{\dagger}\|p(\cdot, \sigma, \cdot)\|_{L^{2}(Q)}^{2}\right), \\
\|G(\sigma, p(\cdot, \sigma))+\alpha p(\cdot, \sigma)\|_{L^{2}(Q)}^{2} \leq 2\left(2 \alpha\|p(\cdot, \sigma)\|_{L^{2}(Q)}^{2}+\|f(\cdot, \sigma, \cdot)\|_{L^{2}(Q)}^{2}\right) .
\end{gathered}
$$

It follows from (2.16)-(2.21) that

$$
\left\|K_{\alpha} p(\cdot, t)\right\|_{L^{2}(Q)}^{2} \leq \tilde{C}_{T}\left\{\|p\|_{L^{2}\left(\mathcal{Q}_{T}\right)}^{2}+\left\|p_{0}\right\|_{L^{2}(Q)}^{2}+\|C\|_{L^{2}\left(\Omega_{T}\right)}^{2}+\|f\|_{L^{2}\left(\mathcal{Q}_{T}\right)}^{2}\right\}
$$

for some constant $\tilde{C}_{T}>0$ depending on $T,\|\beta\|_{L^{\infty}\left(\mathcal{Q}_{T}\right)},\|\mu\|_{L^{\infty}\left(\mathcal{Q}_{T}\right)}, L_{g}$, and $\underline{g}_{T}$. In particular, $K_{\alpha} p \in$ $L^{\infty}\left(0, T ; L^{2}(Q)\right)$ and we find that $K_{\alpha} p \in E_{T,+}$. Next, as usual, we introduce an equivalent norm

$$
\|p\|_{\lambda}:=\operatorname{ess}_{\sup } \operatorname{se}_{t(0, T)} e^{-\lambda t}\|p(\cdot, t, \cdot)\|_{L^{2}(Q)}
$$


in $L^{\infty}\left(0, T ; L^{2}(Q)\right)$ for $\lambda>0$. Let $p_{1}, p_{2} \in E_{T,+}$. Considering $p:=p_{1}-p_{2}$, by linearity, $K_{\alpha} p$ satisfies (2.15) with $p_{0} \equiv 0, C(t, x) \equiv 0, f(s, t, x) \equiv 0$ and hence by $(2.22)$, we have

$$
\begin{aligned}
& e^{-2 \lambda t}\left\|K_{\alpha} p_{1}(\cdot, t)-K_{\alpha} p_{2}(\cdot, t)\right\|_{L^{2}(Q)}^{2} \\
& \quad \leq \tilde{C}_{T} e^{-2 \lambda t} \int_{0}^{t} e^{2 \lambda \sigma} e^{-2 \lambda \sigma}\left\|p_{1}(\cdot, \sigma)-p_{2}(\cdot, \sigma)\right\|_{L^{2}(Q)}^{2} d t \leq \frac{\tilde{C}_{T}}{2 \lambda}\left\|p_{1}-p_{2}\right\|_{\lambda}^{2}
\end{aligned}
$$

This implies that $K_{\alpha}$ is a contraction in $E_{T,+}$ if $\lambda>0$ is taken large enough. Thus, there exists a unique fixed point $p$ in $E_{T,+}$ of $K_{\alpha}$. Since the fixed point $p$ satisfies $(2.22)$ with $K_{\alpha} p(\cdot, t)=p(\cdot, t)$, using Gronwall's lemma, the estimate (2.14) is obtained. This completes the proof.

Corollary 2.5. For any $u \in \mathcal{U}$, there exists a unique mild solution $p=p^{u} \in L^{\infty}\left(0, T ; L^{2}(Q)\right)$ of (1.1) satisfying $p^{u}(s, t, x) \geq 0$ a.e. in $\mathcal{Q}_{T}$.

Proof. Consider $\mu+u$ as the mortality rate $\mu$ in (2.1) and apply Theorem 2.4.

\section{Existence of optimal control}

In this section, we establish the existence of an optimal control which maximizes the optimal harvesting problem (1.2) governed by (1.1). In addition to (H1)-(H3), we impose on the weight function $w(s, t, x)$ the following condition

(H4) $w \in L^{\infty}\left(\mathcal{Q}_{T}\right), w(s, t, x) \geq 0$ a.e. in $\mathcal{Q}_{T}$.

By Corollary 2.5, for any $u \in \mathcal{U}$, there exists a unique mild solution $p^{u} \in L^{\infty}\left(0, T ; L^{2}(Q)\right)$ of $(1.1)$ such that $p^{u}(s, t, x) \geq 0$ a.e. in $\mathcal{Q}_{T}$. Then we have

Theorem 3.1. Let (H1)-(H4) hold. Then the optimal harvesting problem (1.2) governed by (1.1) has at least one optimal solution.

Proof. Define $\Psi: \mathcal{U} \rightarrow \mathbb{R}_{+}$by

$$
\Psi(u)=\int_{\mathcal{Q}_{T}} w(s, t, x) u(s, t, x) p^{u}(s, t, x) d s d t d x
$$

and put $d=\sup _{u \in \mathcal{U}} \Psi(u)$. By (2.14), we find that

$$
\left\|p^{u}(\cdot, t)\right\|_{L^{2}(Q)} \leq C^{\prime}
$$

for some constant $C^{\prime}>0$ independent of $u \in \mathcal{U}$. Hence we have $0 \leq d<\infty$. Let $\left\{u_{N}\right\} \subset \mathcal{U}$ be a sequence satisfying $d-\frac{1}{N}<\Psi\left(u_{N}\right) \leq d$. By (3.1), $\left\|p^{u}\right\|_{L^{2}\left(\mathcal{Q}_{T}\right)}$ is bounded in $u$ and hence there exists a subsequence denoted again by $\left\{u_{N}\right\}$ such that $p^{u_{N}}$ converges weakly to some $p^{*}$ in $L^{2}\left(\mathcal{Q}_{T}\right)$. Using Mazur's theorem (see e.g. [2, p.69]), there exists a sequence $\left\{\widetilde{p}_{N}\right\}$ in $L^{2}\left(\mathcal{Q}_{T}\right)$ satisfying

$$
\begin{aligned}
& \tilde{p}_{N}(s, t, x)=\sum_{i=N+1}^{k_{N}} \lambda_{i}^{N} p^{u_{i}}(s, t, x), \lambda_{i}^{N} \geq 0, \sum_{i=N+1}^{k_{N}} \lambda_{i}^{N}=1\left(k_{N} \geq N+1\right), \\
& \tilde{p}_{N} \rightarrow p^{*} \quad \text { strongly in } L^{2}\left(\mathcal{Q}_{T}\right) .
\end{aligned}
$$

Define the sequence $\left\{\widetilde{u}_{N}\right\}$ by

$$
\widetilde{u}_{N}(s, t, x)= \begin{cases}\frac{\sum_{i=N+1}^{k_{N}} \lambda_{i}^{N} u_{i}(s, t, x) p^{u_{i}}(s, t, x)}{\sum_{i=N+1}^{k_{N}} \lambda_{i}^{N} p^{u_{i}}(s, t, x)}, & \text { if } \sum_{i=N+1}^{k_{N}} \lambda_{i}^{N} p^{u_{i}}(s, t, x) \neq 0, \\ \zeta_{1}(s, t, x), & \text { if } \sum_{i=N+1}^{k_{N}} \lambda_{i}^{N} p^{u_{i}}(s, t, x)=0 .\end{cases}
$$


Then $\widetilde{u}_{N} \in \mathcal{U}$. Since $\left\{\widetilde{u}_{N}\right\}$ is bounded in $L^{2}\left(\mathcal{Q}_{T}\right)$, there exists a subsequence denoted again by $\left\{\widetilde{u}_{N}\right\}$ which converges weakly to some $u^{*}$ in $L^{2}\left(\mathcal{Q}_{T}\right)$. We shall show that this $u^{*}$ gives an optimal control to the optimal harvesting problem (1.2) and (1.1). Note that by linearity, $\widetilde{p}_{N}$ is a mild solution to (1.1) with $\widetilde{u}_{N}$ instead of $u$. Since $\widetilde{p}_{N}$ converges strongly to $p^{*}$ in $L^{2}\left(\mathcal{Q}_{T}\right)$ and $\widetilde{p}_{N} \in L^{\infty}\left(0, T ; L^{2}(Q)\right)$, we find that $p^{*} \in L^{\infty}\left(0, T ; L^{2}(Q)\right)$ and $p^{*}$ is a mild solution of (1.1) for $u=u^{*}$. By uniqueness of solutions, we conclude that $p^{*}=p^{u^{*}}$ and

$$
\begin{aligned}
& \int_{\mathcal{Q}_{T}} w(s, t, x) \widetilde{u}_{N}(s, t, x) \widetilde{p}_{N}(s, t, x) d s d t d x \\
& \rightarrow \int_{\mathcal{Q}_{T}} w(s, t, x) u^{*}(s, t, x) p^{*}(s, t, x) d s d t d x=\Psi\left(u^{*}\right)
\end{aligned}
$$

as $N \rightarrow \infty$. On the other hand,

$$
\begin{aligned}
& \int_{\mathcal{Q}_{T}} w(s, t, x) \widetilde{u}_{N}(s, t, x) \widetilde{p}_{N}(s, t, x) d s d t d x \\
& \quad=\sum_{i=N+1}^{k_{N}} \lambda_{i}^{N} \int_{\mathcal{Q}_{T}} w(s, t, x) u_{i}(s, t, x) p^{u_{i}}(s, t, x) d s d t d x=\sum_{i=N+1}^{k_{N}} \lambda_{i}^{N} \Psi\left(u_{i}\right) \rightarrow d
\end{aligned}
$$

as $N \rightarrow \infty$. Thus we obtain $d=\Psi\left(u^{*}\right)$ and we conclude that $\left(u^{*}, p^{u^{*}}\right)$ is an optimal solution to the optimal harvesting problem (1.2) and (1.1).

\section{Conclusion}

We translated linear size-structured population models with diffusion to abstract partial differential equations in Hilbert space $L^{2}(\Omega)$. Based on the semigroup theory, we introduced a notion of mild solution which has nice properties as it satisfies the equation along characteristic curves. The existence of a unique nonnegative mild solution was shown. We then formulated an optimal harvesting problem to maximize the total price or total harvest through mild solutions and the existence of an optimal harvesting effort was shown.

Acknowledgements. The author thanks anonymous two reviewers for several comments for improvement of this paper. The work was supported by JSPS KAKENHI Grant Number 25400140.

\section{References}

[1] A.S. Ackleh, H.T. Banks, K. Deng. A finite difference approximation for a coupled system of nonlinear size- structured populations. Nonlinear Anal., 50 (2002), 727-748.

[2] S. Anita. Analysis and control of age-dependent population dynamics. Kluwer Acad. Publ., Dordrech, 2000.

[3] A. Calsina, J. Saldaña. A model of physiologically structured population dynamics with a nonlinear individual growth rate. J. Math. Biol., 33 (1995), 335-364.

[4] D. Daners, P. Koch Medina. Abstract evolution equations, periodic problems and applications. Pitman Research Notes in Mathematics Series Vol. 279, Longman Scientific \& Technical, 1992.

[5] A.M. de Roos. A gentle introduction to physiologically structured population models. in Structured-population models in marine, terrestrial, and freshwater systems (S. Tuljapurkar and H. Caswell eds.), Chapman \& Hall, New York, 1996.

[6] J.Z. Farkas, T. Hagen. Stability and regularity results for a size-structured population model. J. Math. Anal. Appl., 328 (2007), 119-136.

[7] N. Hritonenko, Yu. Yatsenko, R. Goetz, A. Xabadia. Optimal harvesting in forestry: steady-state analysis and climate change impact. J. Biological Dynamics, 7 (2012), 41-58.

[8] N. Kato, H. Torikata. Local existence for a general model of size-dependent population dynamics. Abstract Appl. Anal., 2 (1997) 207-226.

[9] N. Kato. Positive global solutions for a general model of size-dependent population dynamics. Abstract Appl. Anal., 5 (2000) 191-206.

[10] N. Kato. A general model of size-dependent population dynamics with nonlinear growth rate. J. Math. Anal. Appl., 297 (2004), 234-256. 
[11] N. Kato. Linear size-structured population models and optimal harvesting problems. J. Ecol. Dev., 5 (2006), No. F06, 6-19.

[12] C. Walker. Some remarks on the asymptotic behavior of the semigroup associated with age-structured diffusive populations. Monatsh Math., 170 (2013), 481-501.

[13] G.F. Webb. Theory of Nonlinear Age-Dependent Population Dyanmics. Marcel Dekker, New York, 1985.

[14] G.F. Webb. Population models structured by age, size, and spatial position. in Structured population models in biology and epidemiology, Lecture Notes in Math. 1936, Springer, Berlin, 2008. 